R E V I S T T A D D E E E S T U D D I O

\title{
Las interfases entre propiedad intelectual y energías renovables
}

\author{
Fernando Alfonso Rivas Mira
}

La teoria supone que los sistemas de propiedad intelectual (IPR) protegen a los inventores y sus invenciones, propiciando al mismo tiempo el desarrollo tecnológico y la satisfacción de los consumidores. En este artículo el autor toma el caso de las energías renovables (ER), y muestra que también intervienen otros factores tales como el grado de desarrollo de los paises, el momento en que tuvo lugar el desarrollo tecnológico, la mentalidad de los consumidores y su preferencia por las energías no renovables (ENR), el uso estratégico de los IPR en los mercados, la situación individual o corporativa de los propios inventores y el papel del Estado. Ilustra su argumentación con referencias al caso de México y presenta un catálogo de patentes obtenidas por inventores mexicanos en materia de energias renovables.

$\mathrm{T}$

eóricamente, las articulaciones entre los derechos de propiedad intelectual (IPR) ${ }^{\mathrm{I}}$ y protección de las invenciones en materia de energías renovables (ER) se remontan a 1883 con el establecimiento del Convenio de París y la expansión de la revolución industrial desde Inglaterra hacia Europa y el resto del mundo. Dicha expansión reveló la permanente necesidad de disponer de energía barata y de transporte fácil, lo que incrementó las invenciones e innovaciones industriales a la vez que la urgencia de protegerlas mediante patentes, que es precisamente el objeto del convenio mencionado.

Los sistemas de IPR fueron objeto de una profunda reformulación en los años noventa del siglo XX. En efecto, los acuerdos conocidos como TRIP², de 1994, estandarizaron los IPR, los volvieron aplicables en prácticamente todo el mundo, y

1 Intellectual Property Rights, siglas reconocidas en la literatura especializada.

2 TRIPS: Trade related Aspects of Intellectual Property Rights, se firmaron en Marrakech el 15 de abril de 1994, en calidad de Anexo C del acta final por la cual se creó la World Trade Organization (Organización Mundial de Comercio) que entró en vigencia el mes de enero de 1995. 
los articularon con el comercio y los negocios. También a finales de ese siglo, la revolución industrial declinaba y era complementada por una economía basada en el conocimiento (Knowledge Basis Economy, KBE). Las energías no renovables (NR) forman parte de la producción de tipo industrial, mientras que las renovables (ER) se perfilan con mayores nexos con el KBE.

Entendemos por ER: «La que, administrada en forma adecuada, puede explotarse ilimitadamente ya que su cantidad disponible no disminuye a medida en que esta se aprovechay. ${ }^{3}$

\section{Los avances tecnológicos han transitado por distintas etapas y momentos.}

Dadas esas premisas, en este trabajo pretendemos contestar a las siguientes preguntas: ¿En qué condiciones históricotecnológicas ocurren las articulaciones entre IPR y ER?, ¿existen diferencias entre los países desarrollados y subdesarrollados? ¿en qué forma los IPR se vuelven estratégicos para la comercialización de invenciones en materia de ER? ¿cuál es la experiencia mexicana al respecto?

En condiciones históricas reales, los avances tecnológicos han transitado por distintas etapas y momentos: la biología, la química, la metalurgia, la electricidad, la electrónica, tuvieron cada una su momento de expansión, que se manifestó entre otros aspectos, en mercancías compradas y vendidas en el mercado a fin de obtener un provecho o beneficio. La competencia por los mercados se expresó también en patentes que protegían de terceros a los propietarios de las invenciones debidamente registradas.

Analizando el tema de la energía en particular, encontramos que aunque la división más amplia y reconocida es entre las renovables o alternativas y las no renovables, en el caso de las primeras encontramos la superposición en el tiempo de la energía mecánica, térmica, química, eléctrica, nuclear, solar, del hidrógeno, biomasa, etc.

Otra clasificación que considera también la superposición en el tiempo distingue entre las ER establecidas o de primera generación, que aquellas cuya tecnología se encuentra muy bien desarrollada, como las grandes centrales hidroeléctricas, y las ER nuevas o de segunda generación, que pese a su gran potencial aún no han sido explotadas, por lo cual son frecuentemente llamadas alternas o no convencionales.

Dentro de las segundas resulta que la energía solar es la más profusa y supera ampliamente a la eólica, geotérmica y oceánica. Por esta razón, cabría esperar que el mayor número de patentes fueran solicitadas y registradas en inventos relacionados precisamente con la energía solar. ${ }^{4}$

3 Secretaría de Energía SENER: «Renovables» en <http://www.sener.gob.mx/wb2/Sener/ Sene_154_renovables $>$, consultado el 17 de marzo de 2006.

4 En la United States Patents and Trade Mark Office USPTO www.uspto.gov,, el número de patentes registradas en materia de energía solar es diez veces mayor que en cualquiera otra de las ER, según datos del National Renewable Energy Laboratory, <http://www.nrel.gov>, Consultado el 17 de enero de 2006. 
Las ER recibieron un primer impulso con la crisis del petróleo de los años setenta. El límite de los recursos petrolíferos fue reconocido mundialmente, y también la necesidad de superar la escasez mediante nuevos desarrollos tecnológicos. Hacia los años noventa, a la conciencia de la escasez se sumó la del daño irreversible al medio ambiente. La teoría de la sustentabilidad demostró que la energía existente no debería ser agotada en perjuicio de las generaciones futuras y que debería ser «limpia», es decir, no contaminante. Para esta teoría tanto la sustentabilidad económica, es decir, la ganancia en los negocios, como la sustentabilidad social y cultural están directamente relacionadas.

\section{Las energías no renovables siguen} siendo las que proporcionan mayores ganancias en los mercados.

Pese a que las energías no renovables siguen siendo las que proporcionan mayores ganancias en los mercados, las renovables compiten con ellas debido a su mayor adecuación, con formas de producir basadas en la innovación, la información y el capital humano (KBE), y con las tendencias a favor de la ecología presentes en la subjetividad colectiva ${ }^{5}$.

En términos de patentabilidad, el nú- mero de solicitudes y registros, así como el valor monetario, es superior en el caso de las no renovables, aunque esos mismos indicadores revelan un crecimiento acelerado de las renovables. A juicio de un experto: «Los resultados indican que la eólica, la solar fotovoltaica e hidráulica son las opciones con mayores perspectivas de crecimiento y participación efectiva, Sin embargo, el logro de estas proyecciones depende de un desarrollo tecnológico factible y competitivo, apoyo decidido del Estado basado en políticas de estímulo y respaldo financiero y la instauración de una cultura energética inspirada en el respeto ambiental, ahorro energético y la sustentabilidad $\rangle^{6}$.

Cabe agregar que al ratificar el Protocolo de Kyoto (1997) los países adquirieron enorme responsabilidad puesto que este instrumento contempla acciones sobre el cambio climático, reducción de los gases de invernadero y acciones públicas encaminadas a la transición hacia energías renovables ${ }^{7}$.

La expansión de las ER ha sido anunciada por el director del National Renewable Energy Laboratory de los Estados Unidos de América: "While humankind has tapped into solar; wind, biomass, and geothermal energy' sources for millennia, science and technology are the keys for making these sources a significant part of the rapidly' growing

5 United Nations, «Energy Services for sustainable development in Asia and the Pacific: policy and practice», Tailandia, Energy' Resources Development Series, № 40, 2005.

6 Posso, Fausto, «Energía y Ambiente, pasado, presente y futuro», Venezuela, Geoenseñanza, Vol 7 2002 , pp. 54 a 73.

7 Estos temas serán convidados en la «Renewable energy 2006 international exhibition» que tendrá lugar en Tokio, Japón en octubre de 2006, <http://www.cnt-inc.co.jp>. 
energy needs of the world in the 21st century. Through the application of physics, chemistry, biology, biotechnology, nanoscience, engineering, and other disciplines come new ways to produce electricity from sum, wind, and the heat of the earth; new, clean transportation fuels from biomass, water; and sunshine; and new plastics, fibers, and materials from plants and wastes - all sustainable, replenishable, and environmentally benign $)^{s}$.

\section{La obtención de ganancias en mercados competitivos es una de las razones más importantes} de las invenciones.

En conclusión, en la medida en que las $\mathrm{ER}$ «encuentren su momento» tanto tecnológico como financiero (rentabilidad) y se expandan en los mercados dado que satisfacen mejor que las no renovables las necesidades reales y culturales de la sociedad, aumentará la necesidad de proteger los IPR sobre esas invenciones, tal como ha sucedido con otros campos tecnológicos y en otros momentos históricos. El sistema de patentes nos recuerda que la obtención de ganancias en mercados competitivos es una de las razones más importantes por las cuales se realizan las invenciones.?

La articulación entre los IPR y las ER depende del grado de desarrollo de los países $^{10}$. En los países desarrollados el porcentaje de utilización de las ER, en relación con el total de energía, es mayor que en los países en vías de desarrollo. Asimismo, la cantidad de solicitudes y registros de IPR también es mayor en los primeros que en los segundos. En los países desarrollados existe una cultura de expectativas respecto de las ER que se convierte en consumo de productos y servicios $y$, en consecuencia, se crea la necesidad de agregar valor a esas mercancías mediante IPR. En cambio en los subdesarrollados lo que más cuenta es el costo de la energía y su capacidad de impulsar los planes de desarrollo. Sin embargo, grupos contestatarios y élites consumidoras de esos países, apoyan el uso de energías limpias o ecológicas. En materia de patentes, el número de solicitudes y registros de patentes nacionales en ER se ha detenido y, por el contrario, las solicitudes de extranjeros se han incrementado. La mayoría de los países en vías de desarrollo experimentan esta misma experiencia ${ }^{11}$.

El carácter público o privado de las fuentes de energía influye en su patentabilidad. Históricamente han ocurrido

8 National Renewable Energy Laboratory, USA, Departamen of Energy, <http://www.nrel.gov>, Consultado el 12 de enero de 2006.

9 Idris, Kamil: «Intellectual Property a power tool for economic growth», World Intellectual Property Organization, Ginebra, 2004, pp. 78

10 Esta distinción ocurre tanto en la teoría como en la práctica, y la literatura suele aludir a ella como la oposición norte-sur. En los paises subdesarrollados habita el $80 \%$ de la población mundial.

1 El Anexo de este trabajo ofrece una lista de invenciones mexicanas en materia de ER. 
transiciones que van del control privado de la energía en el siglo XIX a un control estatal (1930-1990), para volver después, en etapas llamadas neo-liberales, a la propiedad y gestión privadas. Si bien es cierto que el fenómeno afectó principalmente a las energías no renovables (petróleo, carbón), también afectó a las renovables, especialmente el sector eléctrico. Lo cierto es que si las ER se vuelven más importantes, aumentará el debate sobre su estatuto público o privado.

\section{El carácter público o privado de las} fuentes de energía influye en su patentabilidad.

Debido a que las patentes son una forma de monopolio que el Estado otorga a los particulares, su uso aumenta en la medida en que las ER son objeto de apropiación privada. En el caso de la energía, en general, las grandes empresas se ocupan de aquellas que tienen gran valor en el mercado (como es el caso del petróleo, la electricidad, el gas) y prestan mucha atención a la protección de sus invenciones mediante estrategias de IPR. Otros tipos de energía, entre ellas la mayoría de las ER son asunto de las PYME, y de los inventores individuales y artesanales poco preocupados en proteger sus IPR debido a que tienen grandes dificultades para competir en el mercado y acercarse a los consumidores.

La articulación depende también de la transición del inventor individual o artesanal hacia el inventor corporativo vinculado a las grandes empresas y sin excluir figuras de cooperación tales como el «out-sourcing» o la subcontratación entre empresas y personas ${ }^{12}$.

El ciclo ideal tradicional consiste en que los científicos e inventores propongan desarrollos tecnológicos nuevos no incluidos entre los mayores adelantos, y que sean susceptibles de materializar industrialmente para comercializarse en el mercado. La concesión de la patente les permite gozar de derechos exclusivos sobre la invención durante veinte años, recuperar la inversión en capital, tiempo, equipo, mano de obra etc., y continuar desarrollando invenciones. Pueden explotar por sí los derechos exclusivos 0 , como generalmente sucede, otorgar licencias o contratos a cambio de regalías. La sociedad y más concretamente el consumidor, también se benefician pues el avance tecnológico no se detiene y transcurridos los veinte años, la invención se convierte en dominio públi$\mathrm{co}$, enriqueciendo el avance tecnológico ${ }^{13}$.

Actualmente los inventores individuales son superados ampliamente por los departamentos de I\&D (Investigación y Desarrollo) de las grandes empresas. Las instituciones de educación superior (IES) también cuentan con centros y departamentos adecuados para tales fines e, in-

12 Con base en los IPR se acuerda realizar investigaciones conjuntas, financiar ciertos productos o etapas, realizar la invención y mantenerla en reserva por un plazo determinado, mantener colaboración entre instituciones, etc.

13 Al respecto ver el portal de la Organización Mundial de Propiedad Intelectual OMPI: <http:// www.wipo.int>, consultado en enero de 2006. 
cluso, hay organizaciones no gubernamentales (ONG), que pueden financiar proyectos de ER. Esta situación da lugar a que las empresas utilicen el sistema de patentes para fines defensivos, e impidan que otros puedan tener acceso a la invención a menos que se les paguen las licencias correspondientes ${ }^{14}$.

En consecuencia puede concluirse que en materia de fuentes de energía renovables hay más inventores individuales que en las no renovables y que, si resuelven patentar su invención, el sistema ideal funcionará con grandes dificultades frente a un mercado real de patentes cerrado y monopólico.

Los inventores suelen usar estratégicamente el sistema de patentes debido a que estas contienen información técnica de gran valor, la que generalmente se encuentra disponible en bancos de datos en línea $^{15}$. Así, el innovador en ER puede averiguar si hay patentes vigentes y no dilapidar tiempo y recursos, al mismo tiempo que evita infringir derechos de terceros. A partir de la información obtenida se puede solicitar la patente para proteger ciertas características del producto o pro- ceso que podrían ser copiadas por los competidores, para impedir que terceros se aprovechen de la invención ${ }^{16}$.

Cabe recordar que son patentables las invenciones que sean resultado de una actividad inventiva ${ }^{17}$ y tengan aplicación industrial. Se puede obtener la protección bajo patente de productos y procesos. El sistema de patentes permite que el Estado cumpla con su obligación de proteger al consumidor cuyo interés es muy claro: que la energía no falte, que se ofrezca en cantidades suficientes, en formas variadas y a precios accesibles ${ }^{18}$.

\section{Al consumidor le interesa que la energía no falte y que se ofrezca en cantidades suficientes, en formas variadas y a precios accesibles.}

$\mathrm{El}$ inventor en ER puede hacer uso estratégico de las patentes, pero también de otras formas de IPR tales como modelos de utilidad, diseños industriales, secretos comerciales ${ }^{19}$, e incluso certificacioGobernabilidad, Barcelona, España, 2005. dirección del Instituto Mexicano de la Propiedad Industrial (IMPI): <wwwimpi.gob.mx>, con la ventaja de que la consulta es gratuita.

ADIAT: «Práctica de valor, aprovechamiento de patentes para el desarrollo tecnológico», en Prácticas de valor de gestión de tecnología en México. ADIAT y CONACYT, México DF, 2005, capítulo 6, pp. 139-161.

Se entiende por invención toda creación humana que transforma la materia o la energía por el hombre y la satisfacción de sus necesidades.

México, APEC Energy Security Workshop, julio 23, «Energy Security Initiative», 2002, páginas 1 y 2. Se entiende por secreto comercial toda información comercial confidencial que confiera a una empresa una ventaja competitiva. Los secretos comerciales abarcan los secretos industriales o de fabricación y los secretos comerciales. La utilización no autorizada de dicha información por personas distintas del titular se considera práctica desleal y violación del secreto comercial. Según el sistema jurídico, la 
nes del tipo ISO 9000 a 14000 . Además, por la vía del derecho de autor se pueden proteger los libros y artículos, las bases de datos y los conocimientos tradicionales relacionados con la energía renovable. Especial mención merecen los programas de informáticos, que en Estados Unidos pueden protegerse indistintamente mediante patentes o derechos de autor.

\section{La necesidad de proteger $y$} comercializar las invenciones no se dio en forma de patentes por la falta de un mercado nacional para ellas.

Para describir la experiencia mexicana en materia de interfases entre los IPR y la ER se considerarán tres etapas: la etapa pionera, que abarcaría de 1900 a 1979; la etapa de institucionalización, de 1979 a 2005 y los retos a futuro, a partir del año 2006.

En la primera etapa, la investigación en ER no recibió el apoyo del Estado y quedó entregada a investigadores individuales y otros pioneros. La necesidad de protección y comercialización de las in- venciones no se dio en forma de patentes, en gran medida debido a que no existía un mercado nacional para ellas.

La segunda etapa comprende la acción institucionalizadora del Estado mexicano, tanto en materia de energía como en IPR. Respecto de la primera no cabe duda de que las acciones más importantes fueron la creación del Instituto Mexicano del Petróleo IMP y del Instituto de Investigaciones Eléctricas. Materia de IPR lo fue la creación del Instituto Mexicano de la Propiedad Industrial (IMPI), equivalente a las dos anteriores.

En efecto, el IMP se creó el 23 de agosto de $1965^{20}$ y el Instituto de Investigaciones Eléctricas (IIE), el 1 de diciembre de $1975^{21}$. Por otro lado, el Estado mexicano dio pasos avanzados en materia de IPR mediante la promulgación de leyes nuevas y la creación del Instituto Mexicano de la Propiedad Industrial IMPI ${ }^{22}$, así como de la firma de los TRIPS de la Organización Mundial de Comercio (OMC) y de participación en el Foro Asia Pacífico de Cooperación Económica (APEC), organismo al que pertenece el Intellectual Property Rights Experts Group, fundado en 1996, entre cuyos otros

protección de los secretos comerciales forma parte del concepto general de protección contra la competencia desleal o se basa en disposiciones especificas o jurisprudenciales sobre la protección de la información confidencial.

20 En México, el Instituto Mexicano del Petróleo (IMP) es el centro de investigación dedicado al área petrolera, cuyos objetivos principales son realizar investigación y desarrollo tecnológico, y prestar servicios especializados orientados a las necesidades estratégicas y operativas de Petróleos Mexicanos (Pemex). Entrega soluciones integrales innovadoras y desarrolla recursos humanos especializados con un enfoque de calidad, oportunidad y precios competitivos. (http://www.imp.mx/, consultado en marzo de 2006). Es la institución mexicana de investigación que ha logrado registrar mayor número de patentes en los Estados Unidos de América y en México

21 Estrada, Claudio A., «Programa de Trabajo del Director del CIE», México DF, CIE-UNAM, 2004.

22 CIE, «Informe Anual 2005», México, UNAM, 2005, p. 38. 
objetivos se cuenta el de investigar y fortalecer los sistemas de propiedad intelectual. Hasta el momento, el grupo no ha aprobado planes individuales o colectivos, ni resoluciones de sus diversas reuniones que pudieran inducir a las economías miembros a promover invenciones y protegerlas mediante patentes $\mathrm{u}$ otras figuras de propiedad intelectual. Pese a su vínculo con las NR (petróleo y sus derivados, gas y otros), el IMP es la institución que tiene el mayor número de patentes registradas en ER. El IIE ${ }^{23}$ no ha hecho uso de dicho sistema.

Una de las experiencias mexicanas más relevantes y representativas de la tercera etapa de nuestra tipología es el Centro de Investigación en Energía de la Universidad Nacional Autónoma de México. En 1979 se creó el Departamento de Energía Solar como parte del Instituto de Investigaciones en Materiales de dicha universidad, acción institucionalizadora que culminó el 13 de noviembre de 1996 con la creación del Centro de Investigación en Energía (CIE) ${ }^{24}$. Entre los objetivos del Centro se cuenta el de obtener patentes en materia de ER. El análisis de la labor que ha realizado revela que en materia de IPR en 2005 el CIE protegió seis paquetes de software y un libro. Es decir, se siguió la estrategia de otorgar protección mediante el derecho de autor. Asimismo, se obtuvo patente en Estados Unidos, México y Canadá sobre la «reducción dinámica de la capa de mojado durante el desplazamiento de un fluido viscoelástico por un fluido de menor viscosidad» y en el territorio mexicano la patente titulada «Sistema de enfriamiento solar avanzado (ciclo solar-gax)». Además, se solicitó una patente para un «refrigerador solar intermitente para la producción de hielo» ${ }^{25}$.

\section{Parte importante de la patente es su comercialización en el mercado mediante licencias y contratos.}

Parte sustancial de una patente es su comercialización en el mercado mediante licencias y contratos, situación que aún no se presenta en el caso del CIE. Los directivos e investigadores han dejado este tipo de negociaciones en manos de la Dirección General de Asuntos Jurídicos de la UNAM.

El CIE está elaborando un Plan de Acción para la generación Eolo Eléctrica en México. El Programa de las Naciones Unidas para el Desarrollo (PNUD) ha pro-

23 El Instituto de Investigaciones Eléctricas (IIE) es un organismo público descentralizado dotado de personalidad jurídica y patrimonio propio, que tiene carácter cientifico y tecnológico. Sus actividades consisten, principalmente, en llevar a cabo proyectos de investigación aplicada y desarrollo tecnológico para el sector eléctrico. Sus principales clientes son la Comisión Federal de Electricidad (CFE), Luz y Fuerza del Centro (LyFC) y Petróleos Mexicanos (Pemex), así como la industria de manufacturas eléctricas e industrias afines. Ver <http://wwwiie.org. $\mathrm{mx}>$, consultado el 16 de marzo de 2006.

24 La nueva ley fue publicada en el DOF el 27 de junio de 1991. El decreto por el cual se creó el IMPI fue publicado en el DOF del 10 de diciembre de 1993.

25 Al respecto, ver <http://www.apec.org/apec/apec_groups/committees/committee_on_trade/ intellectual_property.html>, consultado el 28 de marzo de 2006. 
porcionado la suma de 4.5 millones de dólares, recursos que se derivarán a un Centro Regional de Investigación y Desarrollo de Tecnologías Eólicas (CERTE), en Oaxaca, $\mathrm{Oax}^{26}$. Además del caso analizado, muestran interfases entre la ER y los IPR, organizaciones no gubernamentales tales como la Asociación Nacional de Energía Solar (ANES) y el Consejo Consultivo para el Fomento de las Energías Renovables, ninguno de los cuales ha solicitado o registrado patentes.

\section{Se requieren políticas públicas que} estimulen la invención en materia de energías renovables.

A partir de 2006, el alza en el costo de las fuentes de energía no renovables, el agotamiento de los pozos de petróleo nacionales y problemas de producción y rentabilidad en materia de electricidad aumentan la presión sobre el mercado. Ello obligará al Estado mexicano a definir leyes y políticas públicas, entre las cuales se cuentan políticas en materia de ER, encaminadas a promover su investigación y protección de las mismas mediante sistemas de IPR, a fin de asegurar rentabilidad y posicionamiento en el mercado global.

\section{CONCLUSIONES}

Las interfases entre la ER y los IPR dependen del desarrollo tecnológico y de la expresión de este en la colocación de productos en el mercado, pues es entonces cuando hay que promover y proteger las invenciones para posicionarse y lograr mayor competitividad. Por esta razón actualmente hay mayor necesidad de patentar las innovaciones en materia de energías no renovables que en las renovables, y entre ellas especialmente la energía solar. Cabe señalar que en el mercado de los países desarrollados se necesitan más patentes en ER que en los países en desarrollo.

El estatuto público o privado de las ER ha evolucionado con el tiempo, $y$ las posibilidades de interfase son mayores cuando el estatuto es privado.

No solo el mercado incide en la interfase, sino también la mentalidad de la sociedad. Al respecto, señalamos que la búsqueda de sustentabilidad expresada en el protocolo de Kyoto propone promover las invenciones en $\mathrm{ER}$, y su comercialización, y, por tanto, la necesidad de recurrir a IPR.

Insistimos en que no solo el mercado y el desarrollo tecnológico son importantes en la interfase, puesto que hay que reconocer el papel de los actores sociales que pueden utilizar las IPR como estrategia para agregar valor a sus productos, posicionarse en el mercado y proteger las invenciones relacionadas con $\mathrm{ER}$.

En el caso de México, en materia de invenciones en energía más que el mercado domina el papel del Estado, lo que se comprueba con la creación del Instituto Mexicano del Petróleo, en el caso de las NR y del Instituto de Investigaciones

26 Estrada, Claudio A., «Programa de Trabajo del Director del CIE», México DF, CIE-UNAM 2004. 
Eléctricas, en el de las ER. La invención privada es escasa. Ambos institutos obtienen patentes tanto nacionales como extranjeras.

El caso del CIE de la UNAM muestra la utilización estratégica de IPR para proteger sus invenciones. Además, ha registrado patentes nacionales y extranjeras, pero no ha logrado comercializarlas, detectándose así una brecha entre inventores, industriales y comercializadores.

En general, se requieren políticas públicas que animen la invención en materia de ER y su protección mediante IPR, para evitar el déficit de energías no renovables y salvar la brecha que separa a México de los países desarrollados.

El Anexo ilustra aspectos relevantes del presente trabajo.

\section{Anexo 1}

Inventores mexicanos en materia de energías renovables, por orden alfabético, año y número de patente o modelo de utilidad registrada por el Instituto Mexicano de la propiedad industrial IMPI 1980-200027.

\section{Aguirre Gandara Manuel, UNAM}

Dispositivo disipador de energía pat: 172240,1993

\section{Beristain, Juárez Felipe}

Instalación para secar productos con el aprovechamiento de energía solar pat: 159392, 1989
3. Bishop, Norman G.; Viramontes, Brown Ricardo

Hylsa, S. A. de C. V. proler

Environmental services, inc.

Método y aparato para la gasificación de desperdicios orgánicos

pat: 191160, 1999

4. Cañedo Ramirez, Roberto

Mejoras a surtidor o calentador de agua a base de energía solar

pat: 160678,1990

5. Chico, Eduardo

Dispositivo concentrador de la energía solar

pat: 164134,1992

6. Mendoza Gómez, Julio Gabriel

Mejoras en relojes solares

pat: 140865,1980

7. Najar; Enrique Rodrigo

Instituto de Investigaciones Eléctricas

Mejoras en calentador geotérmico y método geotérmico de calentamiento de fluidos pat: 160406,1990

8. Diez de Sollano y Ortega, Carlos Mejoras en calentador solar pat: 141774,1980

9. Diez de Sollano y Ortega, Carlos Mejoras en molino de viento para el aprovechamiento de la energía eólica pat: 157331,1988 
10. Gutiérrez Martínez, Filiberto Calentador solar con circulación radial pat: 171846,1993

\section{Gutiérrez Martinez, Filiberto}

Calentador solar con recirculación autopropulsada del líquido entre el propio calentador y un intercambiador de calor situado a nivel inferior pat: 192116,1999

\section{Hendrichs Troeglen, Nicolás}

Modelo de utilidad de elemento de captación de energía solar inmerso en el líquido por calentar en colectores planos o charolas mu: 000282, 1995

13. Hernández Galán, José Luis; Sánchez Uribe, Severiano

Instituto de Investigaciones Eléctricas Mejoras en un procedimiento y equipo anticontaminante para la generación de biogas mediante la digestión anaerobia de estiércol vacuno y otros desechos orgánicos ci: 005429,1983

\section{Hernández Mojica, Tonatiuh}

Mejoras a captador almacenador de calor por medio de energía solar pat: 162917,1991

15. Lecanda Chavero, Alberto Ernesto Mejoras en calentador solar pat: 151700,1985

16. Martinez del Río Lozano, Eustaquio Mejoras en calentador solar pat: 153048,1986
17. Ochoa Altamirano, José Eduardo Estructura rodante para la conversión de energía de olas a eléctrica pat: 194945,2000

\section{Ochoa Altamirano, José Eduardo} Chasis rodante para transferencia de energía de olas a eléctrica pat: 181407,1996

\section{Olechnowicz Fridman, Elias}

Mejoras en un sistema solar para calentamiento de fluidos

pat: 190950, 1999

\section{Osorio Saucedo, Ruperto}

Nueva caja de transmisión activada por el viento para subir agua

pat: 198571,2000

\section{Osorio Saucedo, Ruperto}

Nueva hélice multipaletas para el aprovechamiento del viento pat: 195155,2000

\section{Ramirez Garcia, Teófilo}

Receptor de radiación para un concentrador solar parabólico

mu: 000601, 1998

\section{Rodríguez Jiménez, Alejandro}

Bio reactor anaeróbio integrado pat: 000511,1996

24. Rodríguez Maciel, Luis Arturo Depósito de agua caliente mejorado para colector solar

pat: 176014,1994 
25. Romero Robles, Victor Manuel

Mejoras en calentador solar pat: 142435,1980

26. Ruiz Rentaria, Francisco Gerardo; Alatorre Mendieta, Miguel Ángel; Merino Ibarra, Martin, UNAM

Dispositivo amplificador y rectificador de oleaje

pat: 179369,1995

\section{Siller Franco, José Luis}

Dispositivo mejorado para aprovechar la energía de la olas para la generación de energía eléctrica, hidráulica y otras pat: 152132,1985

\section{Siller Franco, José Luis}

Mejoras en sistema de hélices o turbinas para la captación o transformación de la energía cinética presente en ríos y corrientes submarinas

pat: 155343,1988

29. Torres Robles, Rafael; Rodriguez Rodriguez, Javier Esteban, IMP

Equipo vaporizador-calentador multietapas para transferir la energía de salmueras geotérmicas a un fluido de trabajo pat: 162761,1991

\section{Torres Robles, Rafael; Rodriguez} Rodriguez, IMP

Mejoras en aparato la determinación de equilibrio líquido-líquido-vapor en un sistema

pat: 159041,1989

31. Torres Robles, Rafael; Rodriguez Rodriguez, IMP
Mejoras al proceso para la generación de potencia a partir de la energía de salmueras geotérmicas

pat: 158282, 1989

\section{Torres Robles, Rafael; Rodriguez} Rodríguez, IMP

Equipo para la transferencia de la energía de salmueras geotérmicas a un fluido de trabajo en procesos para la generación de potencia

pat: 168569,1993

\section{Bibliografía}

APEC Energy Security Workshop, México, 23 de julio de 2002: «Energy Security Initiative».

Asociación Mexicana de Directivos en Investigación Aplicada y Desarrollo (ADIAT), «Práctica de valor, aprovechamiento de patentes para el desarrollo tecnológicon en Prácticas de valor de gestión de tecnologia en México, ADIAT y CONACYT, México DF, 2005.

Centro de Investigación en Energía (CIE): Informe Anual 2005, México, UNAM, 2005.

Estrada, Claudio A., «Programa de Trabajo del Director del CIE», México DF, CIE-UNAM, 2004.

Idris, Kamil, «Intellectual Property: a power tool for economic growth», World Intellectual Property Organization, Ginebra, 2004.

Jacobkis, David, «Las patentes de software, un obstáculo para la innovación» en Boletin Gobernabilidad, Barcelona, España, 2005.

Naciones Unidas: «Energy Services for sustainable development in Asia and the Pacific: policy and practice», Tailandia, Energy Resources Development Series, $N^{0} 40,2005$.

Posso, Fausto, «Energía y Ambiente, pasado, presente y futuro", Caracas, Geoenseñanza, Vol. 7,2002.

Secretaria de Energia (SENER), «Energías Renovables para el Desarrollo Sustentable en Méxicon, México, 2006. 
Sitios o portales

Instituto Mexicano de la Propiedad Industrial (IMIPI), http://www.impi.gob.mx

Secretaria de Energía (SENER): «Renovables», en http://www.sener.gob.mx

United States Patents and Trade Mark Office (USPTO) www.uspto.gov

National Renewable Energy Laboratory, http:// www.nrel.gov

Renewable Energy 2006 International Exhibition. http://www.ent-inc.co.jp
Organización Mundial de Propiedad Intelectual (OMPI) http://www.wipo.int

Instituto de Investigaciones Eléctricas (IIE): http:/ /wnww.iie.org.mx

Instituto Mexicano del Petróleo (IMP): http:// www.imp.mx/

APEC Intellectual Property Rigths Experts Group: http://www.apec.org/apec/apec_groups/ committees/committee_on_trade/ intellectual_property.html 